\title{
Subject Evaluability Criteria and Classification Document
}

National Cancer Institute

\section{Source}

National Cancer Institute. Subject Evaluability Criteria and Classification Document. NCI

Thesaurus. Code C115735.

Records detailing clearly defined criteria, which are used to evaluate and assign study subjects to a population group, established by a statistical analysis plan (SAP). 